\title{
ANÁLISIS MULTITEMPORAL DE LOS INDICADORES DE CALIDAD DE AGUA EN CORRIENTES SUPERFICIALES (ICA) DE LA CUENCA ALTA DEL RÍO BOGotá (COLOMbia)
}

\author{
Andrea Cecilia Sanabria-Suárez ${ }^{1}$, Carlos Montenegro-Marín², Mario \\ Fernando Castro-Fernández ${ }^{3}$, Daissy Milena Díaz-Casallas ${ }^{4}$ \\ ${ }^{1}$ Estudiante de Administración Ambiental, Universidad Distrital Francisco José de Caldas, Bogotá, Colombia \\ ${ }^{2}$ Docente investigador, Universidad Distrital Francisco José de Caldas, Universidad Cooperativa de Colombia, \\ Bogotá, Colombia \\ ${ }^{3}$ Docente investigador, Universidad Cooperativa de Colombia, Bogotá, Colombia. Correo electrónico: mario. \\ castrof@campusucc.edu.co \\ ${ }^{4}$ Estudiante Maestría en Desarrollo Sustentable y Gestión Ambiental, Universidad Distrital Francisco José de \\ Caldas, Bogotá, Colombia
}

Fecha de recibido: 20 de noviembre del 2016 Fecha de aprobado: 27 de febrero del 2017

Cómo citar este artículo: A. C. Sanabria-Suárez, C. Montenegro-Marín, M. F. Castro-Fernández, D. M. Díaz-Casallas, "Análisis multitemporal de los indicadores de calidad de agua en corrientes superficiales (ICA) de la cuenca alta del Río Bogotá (Colombia)", Ingeniería Solidaria, vol. 13, n. ${ }^{0}$ 22, mayo de 2017, pp. 39-54. doi: http://dx.doi.org/10.16925/in.v13i22.1751

Resumen. Introducción: este artículo presenta las variaciones en la calidad del agua en la cuenca alta del río Bogotá, mediante la interpretación de los resultados obtenidos en monitoreos semestrales realizados durante el periodo 2008-2015 por la Corporación Autónoma Regional de Cundinamarca (CAR). Estos resultados previos corresponden al proyecto de investigación Variables económicas, sociales e institucionales de mayor influencia en los indicadores de segunda generación, relacionados con el recurso hídrico y su gestión, desarrollado por el grupo de investigación Neotic de la Facultad de Ingeniería de la Universidad Cooperativa de Colombia, sede Bogotá, durante el 2016. Metodología: a partir de la información suministrada por la CAR, se calculó el índice de calidad de agua (ICA) establecido por el Instituto de Hidrología, Meteorología y Estudios Ambientales (Ideam) para Colombia. Resultados: el uso excesivo de agroquímicos en la cuenca alta del río Bogotá es un aspecto con repercusiones negativas para este, pues desde la cuenca alta ya comienza a evidenciar deterioro en su calidad. Los factores antrópicos son la principal causa de contaminación y deterioro ambiental por las actividades agroindustriales que se desarrollan en torno a sus aguas. Conclusiones: si bien las alcaldías municipales están al tanto de la problemática en torno al río Bogotá e incluyen dentro de sus planes de desarrollo municipales mecanismos para atenderlas, los resultados obtenidos evidencian la necesidad de formular nuevas estrategias ambientales o reformular las existentes para el cuidado y protección del recurso.

Palabras clave: análisis multitemporal, calidad de agua, índice ICA, recurso hídrico. 


\title{
Multitemporal Analysis of The Water Quality INDEX (WQI) IN SURFACE CURRENTS IN THE UPPER BASIN OF THE Bogotá River (COLOMBia)
}

\begin{abstract}
Introduction: This article presents the variations in water quality in the upper basin of the Bogotá River by interpreting the results obtained from the semiannual monitoring conducted by the Cundinamarca environmental authority (Corporación Autónoma Regional - CAR) during 2008-2015. These results correspond to the research project Economic, social and institutional variables of greater influence over second generation indicators related to the water resource and its management, carried out by the research group Neotic of the School of Engineering, Universidad Cooperativa de Colombia, Bogotá, during 2016. Method: Based on the information provided by CAR, the water quality index (WQI) established by the Institute of Hydrology, Meteorology and Environmental Studies (IDEAM) for Colombia was calculated. Results: The excessive use of agrochemicals in the upper basin of the Bogotá River has repercussions on it, since water quality already shows deterioration from this place. Anthropic factors are the main cause of pollution and environmental deterioration due to agroindustrial operations carried out around its waters. Conclusions: Although municipal mayors are aware of the problems of the Bogotá River and include mechanisms to address them in their municipal development plans, the results obtained demonstrate the need to formulate new environmental strategies or reformulate existing ones for the care and protection of this resource.
\end{abstract}

Keywords: multitemporal analysis, water quality, WQI, water resource.

\section{ANÁlise MULTITEMPORAL DOS INDICADORES DE QUALIDADE DE ÁGUA EM CORRENTES SUPERFICIAIS (ICA) DA BACIA Alta do Rio Bogotá (Colômbia)}

Introdução: este artigo apresenta as variações na qualidade da água na bacia alta do rio Bogotá, mediante a interpretação dos resultados obtidos em monitoramentos semestrais realizados durante o período 2008-2015 pela Corporación Autónoma Regional de Cundinamarca (CAR). Esses resultados prévios correspondem ao projeto de pesquisa "Variáveis econômicas, sociais e institucionais de maior influência nos indicadores de segunda geração, relacionados com o recurso hídrico e sua gestão", desenvolvido pelo grupo de pesquisa Neotic, da Faculdade de Engenharia da Universidad Cooperativa de Colombia, sede Bogotá, durante o ano de 2016. Metodologia: a partir da informação fornecida pela CAR, calculou-se o índice de qualidade da água (ICA), estabelecido pelo Instituto de Hidrologia, Meteorologia e Estudos Ambientais (Ideam) para a Colômbia. Resultados: o uso excessivo de agroquímicos na bacia alta do rio Bogotá é um aspecto com consequências negativas para este, pois, desde a bacia alta, já começa a evidenciar deterioração em sua qualidade. Os fatores antrópicos são a principal causa de poluição e deterioração ambiental devido às atividades agroindustriais que se desenvolvem em torno de suas águas. Conclusões: embora as prefeituras municipais estejam cientes da problemática sobre o rio Bogotá e incluam em seus planos de desenvolvimento municipal mecanismos para atendê-las, os resultados obtidos evidenciam a necessidade de formular novas estratégias ambientais ou reformular as existentes para o cuidado e a proteção do recurso.

Palavras-chave: análise multitemporal, ICA, qualidade da água, recurso hídrico. 


\section{Introducción}

Según el Informe de las Naciones Unidas sobre el Desarrollo de los Recursos Hídricos en el Mundo 2016: Agua y Empleo [1], las dinámicas de la población y el estándar de vida han impulsado la producción y el consumo de bienes y servicios, a fin de satisfacer las necesidades cada vez mayores de una población creciente y más solvente económicamente. La disponibilidad de agua también depende en gran medida de la calidad del agua, pues la que es de mala calidad no es apta para varios usos y el costo del tratamiento puede ser prohibitivo, agravando así la carga de su escasez económica. Según un estudio realizado por Veolia y el IFPRI (2015, p. 3): "se prevé que el deterioro de la calidad del agua aumente rápidamente en los próximos decenios, lo que, a su vez, aumentará los riesgos para la salud humana, el desarrollo económico y los ecosistemas", citado por [1].

La calidad del agua se mide de acuerdo con distintos parámetros, mediante los cuales se cuantifica el grado de alteración de las cualidades naturales y se la clasifica para un uso determinado [2]. La dinámica natural de las corrientes de agua superficiales se deriva de un comportamiento determinado por variaciones biológicas y físico-químicas que ejercen presión sobre el cuerpo de agua [3].

El ICA se ha convertido en un instrumento fundamental para transmitir información sobre la calidad del recurso hídrico a las autoridades competentes y al público en general. Es un indicador compuesto que integra información de varios parámetros de calidad del agua y presenta diferentes metodologías, según su autor. Este índice es una herramienta matemática para la calidad y puede ser utilizado con el fin de transformar grandes cantidades de datos sobre la calidad del agua en una escala de medición única [2].

Como se mencionó previamente en el artículo "Indicadores de la calidad del agua: evolución y tendencias a nivel global" [2], si se revisa el trabajo de Lumb, Halliwell y Sharma, se observa que los intentos de clasificar el agua de acuerdo con su grado de pureza se remontan a mediados del siglo $\mathrm{xx}$ con los estudios de Horton en la década de los sesenta, y los de Landwehr en la de los setenta. Más adelante, Ott y Steinhart revisaron más de veinte índices de calidad del agua que fueron utilizados hasta finales de la década de los setenta. Desde 1965, cuando Horton propuso el primer índice de calidad del agua, una gran cantidad de consideraciones han surgido en torno al desarrollo de métodos de índice. Adicionalmente, en Colombia algunos autores estudiaron cómo el cálculo de índices de calidad de agua no es una actividad tan rutinaria como se cree, aunque se aplican regularmente en la industria del petróleo y algunas corporaciones autónomas regionales en Bogotá, Barranquilla, Bucaramanga, Cali y Manizales, al estimar los ICA e ICO en sus programas de monitoreo.

El estado de calidad de agua en las corrientes superficiales es el panorama ideal para la formulación de propuestas, políticas o normativas, determinantes para una adecuada gestión del recurso hídrico. Para tal efecto, el Sistema Nacional Ambiental (sINA), organismo responsable del cuidado del medio ambiente y los recursos naturales, por intermedio de uno de sus integrantes, el Ideam, responsable del Sistema de Información Ambiental de Colombia (sIAC), integrado entre otros por el Sistema de Información del Recurso Hídrico (SIRH), trabaja de manera articulada con las autoridades ambientales regionales, urbanas y el Sistema de Parques Nacionales, con el propósito de hacer control, seguimiento y registro de la información hídrica reportada en torno a cuatro temáticas: oferta, demanda, calidad y gestión del recurso.

La gestión ambiental de las fuentes hídricas en Colombia está a cargo de las Corporaciones Autónomas Regionales de cada zona del país, las cuales realizan monitoreos de calidad hídrica e implementan metodologías de cálculo de indicadores y estrategias. Tal como lo expresa Peña [4], se desarrollaron a partir de la década de los sesenta, momento desde el cual estas entidades velan por la atención de alcances y objetivos nacidos de movimientos ecológicos mundiales y la legislación ambiental, en aras del cuidado, la preservación, la protección y la defensa de los recursos naturales de la nación. A partir de los años sesenta se evidencia el establecimiento de estrategias para la valoración de calidad de aguas, cuya principal herramienta son las redes de monitoreo que precisan la condición del recurso hídrico en una fuente natural.

Se efectuó una labor exploratoria con el Grupo de Investigación e Interconexión de Redes Académicas (GIIRA), de la Facultad de Ingeniería de la Universidad Distrital Francisco José de Caldas, de Bogotá, la cual consistió en la limpieza y organización de los datos correspondientes a los parámetros físico-químicos del agua del río Bogotá, 
de manera que permitiera el cálculo del ICA, de acuerdo con la hoja metodológica establecida por el Ideam.

El análisis que a continuación se presenta se centra en la cuenca alta del río Bogotá, en el periodo 2008-2015. El territorio inicia en el Páramo de Guacheneque, estación de monitoreo Aguas Arriba Villapinzón, hasta la estación de monitoreo Río Teusacá, ubicada en el municipio de Zipaquirá.

\section{2. Área de estudio}

El río Bogotá es uno de los afluentes más importantes a nivel nacional, con una extensión de 375 kilómetros desde su nacimiento en Villapinzón, hasta su desembocadura en el río Magdalena.

El río atraviesa toda la zona centro del país, exactamente en el departamento de Cundinamarca, y recorre los municipios de Villapinzón, Chocontá, Suesca, Sesquilé, Nemocón, Tocancipá, Zipaquirá, Cajicá, Cota, Funza, Soacha, San Antonio de Tequendama, El Colegio, La Mesa, Anapoima, Apulo, Tocaima, Agua de Dios, Ricaurte y Girardot. La cuenca del río Bogotá hace parte de la Macrocuenca Magdalena-Cauca, columna vertebral de toda la fuente hídrica, como se observa en la figura 1.

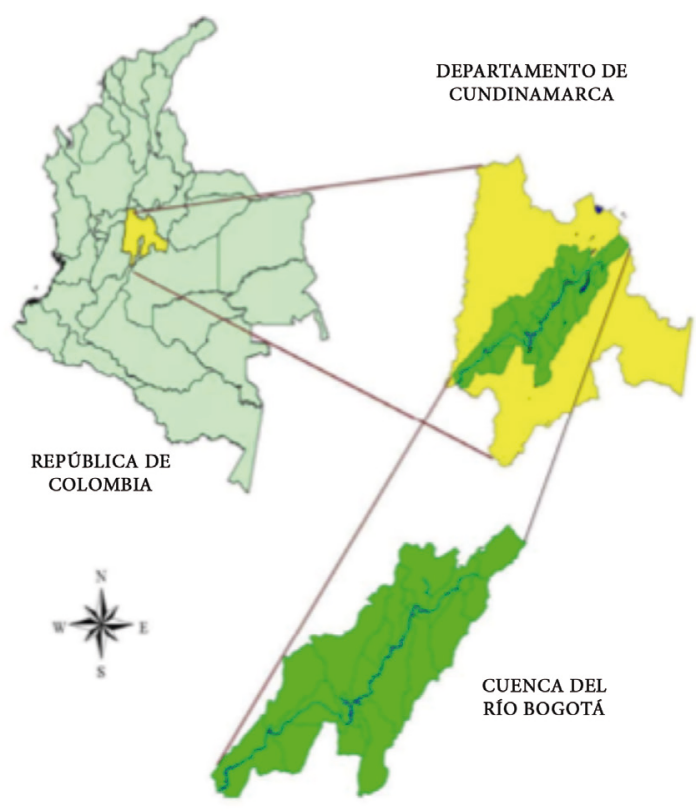

Figura 1. Ubicación geográfica del río Bogotá Fuente: [5]

\section{Metodología}

\subsection{Obtención de datos}

Por medio de solicitud directa al Laboratorio de Calidad Ambiental de la CAR, se logra acceder a los resultados de monitoreo de calidad del agua de la cuenca del río Bogotá correspondiente al periodo 2008-2015. La cuenca alta del río Bogotá cuenta con 31 puntos de monitoreo, ubicados estratégicamente desde el nacimiento del río en el páramo de Guacheneque, en el municipio de Villapinzón, hasta el punto denominado río Teusacá, ubicado en el municipio de Zipaquirá. Estos puntos de monitoreo han sido dispuestos por la Corporación Autónoma Regional, ente encargado del seguimiento de la fuente hídrica.

Villapinzón, Chocontá, Suesca, Gachancipá, Tocancipá y Zipaquirá, son los nombres de los municipios del departamento de Cundinamarca que pertenecen a la cuenca alta del río Bogotá. Los puntos de monitoreo dispuestos en los diferentes segmentos del río varían en cantidad para cada municipio, y se establecen de acuerdo con la intencionalidad del análisis realizado por la CAR.

La clasificación de las estaciones de monitoreo por municipios se realizó teniendo en cuenta los datos de coordenadas geográficas para cada punto de monitoreo. Los datos originales se tomaron de los boletines de Calidad Hídrica de la CAR para el año 2013. Los 31 puntos de monitoreo de la cuenca alta del río se presentan en la tabla 1.

Tabla 1. Estaciones de monitoreo por municipio

\begin{tabular}{|l|l|l|}
\hline $\begin{array}{c}\text { Tipo de } \\
\text { fuente }\end{array}$ & Nombre punto de monitoreo & Municipio \\
\hline Principal & Aguas arriba Villapinzón & Villapinzón \\
\hline Principal & Puente de Villapinzón & Villapinzón \\
\hline Principal & Aguas arriba Q. Quincha & Villapinzón \\
\hline Afluente & Q. Quincha & Villapinzón \\
\hline Principal & Estación Chingacio & Chocontá \\
\hline Afluente & Agregados Chocontá & Chocontá \\
\hline Afluente & Río Tejar & Chocontá \\
\hline Principal & Puente vía Telecom & Chocontá \\
\hline Afluente & $\begin{array}{l}\text { Descarga municipio de } \\
\text { Chocontá }\end{array}$ & Chocontá \\
\hline Principal & $\begin{array}{l}\text { Aguas abajo municipio de } \\
\text { Chocontá }\end{array}$ & Chocontá \\
\hline
\end{tabular}

Continúa en la pág. 45 
Viene de la pág. 44

\begin{tabular}{|l|l|l|}
\hline $\begin{array}{c}\text { Tipo de } \\
\text { fuente }\end{array}$ & Nombre punto de monitoreo & Municipio \\
\hline Principal & Estación LG Saucio & Chocontá \\
\hline Afluente & Descarga embalse del Sisga & Chocontá \\
\hline Principal & Estación LM Santa Rosita & Suesca \\
\hline Principal & Puente Santander & Suesca \\
\hline Afluente & Descarga municipio Suesca & Suesca \\
\hline Principal & $\begin{array}{l}\text { Aguas abajo municipio de } \\
\text { Suesca }\end{array}$ & Suesca \\
\hline Afluente & $\begin{array}{l}\text { Descarga embalse de } \\
\text { Tominé }\end{array}$ & Suesca \\
\hline Principal & $\begin{array}{l}\text { Aguas arriba descarga } \\
\text { Papeles y Molinos }\end{array}$ & Suesca \\
\hline Principal & Estación LG-PTE Florencia & Gachancipá \\
\hline Afluente & $\begin{array}{l}\text { Descarga municipio } \\
\text { Gachancipá }\end{array}$ & Gachancipá \\
\hline Principal & $\begin{array}{l}\text { Aguas abajo municipio } \\
\text { Gachancipá }\end{array}$ & Gachancipá \\
\hline Principal & Estación LM- Tocancipá & Tocancipá \\
\hline Afluente & $\begin{array}{l}\text { Descarga municipio } \\
\text { Tocancipá }\end{array}$ & Tocancipá \\
\hline Principal & Aguas arriba Termozipa & Tocancipá \\
\hline Afluente & Descarga Termozipa & Tocancipá \\
\hline Principal & Hacienda El Triunfo & Zipaquirá \\
\hline Afluente & Río Neusa & Zipaquirá \\
\hline Principal & Estación LG- El Espino & Zipaquirá \\
\hline Afluente & Río Negro & Zipaquirá \\
\hline Principal & Aguas abajo Río Negro \\
\hline Afluente & Río Teusacá & Zipaqúa \\
\hline
\end{tabular}

Fuente: elaboración propia

\subsection{Calculo índice de calidad del agua}

El índice de calidad del agua es el valor numérico que califica, en una de cinco categorías, la calidad del agua de una corriente superficial, con base en las mediciones obtenidas para un conjunto de cinco o seis variables, registradas en una estación de monitoreo $j$ en el tiempo $t$. Los valores optativos que puede llegar a tomar el indicador han sido clasificados en categorías; de acuerdo con ellos se califica la calidad del agua de las corrientes superficiales, al cual se le ha asociado un color como señal de alerta [6].

La hoja metodológica del indicador desarrollada por el Ideam [6] establece el método para el cálculo del ICA, a partir de los datos de concentración de un conjunto de cinco o seis variables, que determinan, en gran parte, la calidad de las aguas corrientes superficiales. La ecuación 1 responde al cálculo del índice de calidad de aguas superficiales.

$$
I C A_{n j t}=\sum_{i=1}^{n} W_{i}^{*} I_{i k j t}
$$

Donde: $I C A_{n j t}$ es el índice de calidad del agua de una determinada corriente superficial en la estación de monitoreo de la calidad del agua $j$ en el tiempo $t$, evaluado con base en $n$ variables. $W_{i}$ es el ponderador o peso relativo asignado a la variable de calidad $i$. Y por su parte, $I_{i k j t}$ es el valor calculado de la variable $i$ (obtenido de aplicar la curva funcional o ecuación correspondiente), en la estación de monitoreo $j$, registrado durante la medición realizada en el trimestre $k$, del período de tiempo $t$. Por último, es el número de variables de calidad involucradas en el cálculo del indicador; en nuestro caso, es $n$ es igual a 5 .

En la tabla 2 se registra la relación entre valores y calificación.

Tabla 2. Índice de calidad de agua ICA

\begin{tabular}{|l|l|l|}
\hline $\begin{array}{c}\text { Categoría } \\
\text { de valores }\end{array}$ & $\begin{array}{c}\text { Calificación de la } \\
\text { calidad del agua }\end{array}$ & \multicolumn{1}{|c|}{$\begin{array}{c}\text { Clasificación } \\
\text { por color }\end{array}$} \\
\hline $0,0-0,25$ & Muy mala & Rojo \\
\hline $0,26-0,50$ & Mala & Naranja \\
\hline $0,51-0,70$ & Regular & Amarillo \\
\hline $0,71-0,90$ & Aceptable & Verde \\
\hline $0,91-1,00$ & Buena & Azul \\
\hline
\end{tabular}

Fuente: [6]

A partir de la recopilación de los resultados de monitoreo de calidad hídrica del río Bogotá, se procedió con la conversión del formato de documentos portátiles (PDF) a un formato de texto plano, con el fin de facilitar su introducción en el motor de base de datos MysQL, para realizar el cálculo del ICA. Una vez obtenidos los resultados, se prosiguió a identificar la categoría punto por punto en los quince (15) semestres de estudio, a partir de la información establecida en la tabla 2, y una vez identificadas las categorías, se graficó la información teniendo en cuenta la clasificación municipal. 
Tabla 3. Valor ICA semestral para el periodo 2008-2015

\begin{tabular}{|c|c|c|c|c|c|c|c|c|c|c|c|c|c|c|c|}
\hline $\begin{array}{c}\text { Punto } \\
\text { N. }^{\circ}\end{array}$ & 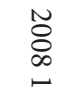 & $\begin{array}{l}N \\
0 \\
0 \\
\infty \\
N\end{array}$ & 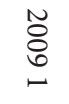 & $\begin{array}{l}\tilde{O} \\
8 \\
0 \\
N\end{array}$ & 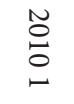 & 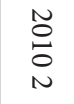 & $\begin{array}{l}\stackrel{\curvearrowright}{\varrho} \\
\sqsupseteq \\
\curvearrowleft\end{array}$ & $\begin{array}{l}\stackrel{N}{\ominus} \\
\stackrel{\Xi}{N}\end{array}$ & 苍 & 古 & 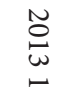 & $\begin{array}{l}\text { No } \\
\stackrel{\omega}{\omega} \\
N\end{array}$ & $\begin{array}{l}\stackrel{N}{\circ} \\
\stackrel{D}{\perp}\end{array}$ & $\begin{array}{l}\stackrel{N}{O} \\
\stackrel{\vec{A}}{N}\end{array}$ & $\begin{array}{l}\text { No } \\
\stackrel{0}{u} \\
\sim\end{array}$ \\
\hline 1 & 0,79 & 0,78 & 0,47 & 0,54 & & & 0,54 & & 0,79 & 0,75 & 0,55 & & & 0,53 & 0,6 \\
\hline 2 & 0,55 & 0,73 & 0,7 & 0,76 & & & 0,68 & & 0,78 & 0,76 & 0,76 & & 0,62 & 0,74 & 0,79 \\
\hline 3 & 0,74 & 0,73 & 0,69 & 0,6 & & & 0,68 & & 0,73 & 0,66 & 0,72 & & 0,62 & 0,75 & 0,78 \\
\hline 4 & 0,57 & 0,65 & 0,59 & 0,62 & & & 0,78 & & 0,71 & 0,69 & 0,55 & & 0,64 & 0,54 & 0,8 \\
\hline 5 & 0,46 & 0,51 & 0,49 & 0,62 & & 0,64 & 0,62 & & & 0,5 & 0,66 & & 0,63 & 0,62 & 0,58 \\
\hline 6 & 0,57 & 0,68 & 0,41 & 0,5 & & 0,47 & 0,62 & & 0,55 & 0,54 & 0,53 & & & 0,5 & 0,57 \\
\hline 7 & 0,69 & 0,72 & & 0,74 & & 0,72 & 0,58 & & 0,77 & & 0,8 & & & 0,79 & 0,75 \\
\hline 8 & 0,44 & 0,6 & 0,46 & 0,63 & 0,52 & 0,66 & 0,73 & & 0,48 & & 0,59 & 0,68 & & 0,74 & 0,47 \\
\hline 9 & 0,34 & 0,41 & 0,42 & 0,41 & 0,38 & 0,39 & 0,43 & & 0,44 & & 0,45 & 0,49 & 0,33 & 0,41 & 0,18 \\
\hline 10 & 0,51 & 0,56 & 0,51 & 0,45 & 0,47 & 0,68 & 0,45 & & 0,48 & & 0,59 & 0,63 & 0,6 & 0,63 & 0,47 \\
\hline 11 & 0,41 & 0,49 & 0,58 & 0,52 & 0,47 & 0,56 & 0,68 & & 0,44 & & 0,71 & 0,62 & 0,62 & 0,57 & 0,52 \\
\hline 12 & 0,79 & 0,6 & 0,75 & 0,63 & 0,79 & & 0,55 & & 0,79 & & & 0,77 & 0,78 & 0,79 & 0,79 \\
\hline 13 & 0,69 & 0,48 & 0,68 & 0,69 & 0,51 & 0,56 & 0,73 & & & & 0,72 & 0,7 & 0,67 & 0,68 & 0,68 \\
\hline 14 & 0,71 & 0,58 & 0,67 & 0,74 & 0,72 & 0,52 & 0,74 & & & & 0,72 & 0,66 & 0,69 & 0,71 & \\
\hline 15 & 0,27 & 0,33 & 0,63 & 0,18 & 0,25 & 0,93 & 0,36 & & & & 0,37 & 0,25 & 0,3 & 0,25 & 0,31 \\
\hline 16 & 0,76 & 0,61 & 0,71 & 0,7 & 0,73 & 0,51 & 0,72 & & & & 0,64 & 0,67 & 0,62 & & 0,62 \\
\hline 17 & 0,78 & 0,71 & 0,74 & 0,78 & 0,58 & & & & & & 0,74 & 0,78 & 0,57 & 0,78 & 0,7 \\
\hline 18 & 0,74 & 0,63 & 0,69 & 0,71 & 0,77 & 0,53 & 0,77 & & & & & 0,73 & 0,71 & 0,72 & 0,69 \\
\hline 19 & 0,76 & 0,61 & 0,69 & 0,74 & 0,77 & 0,55 & 0,77 & & 0,6 & & 0,66 & 0,65 & 0,7 & 0,62 & 0,76 \\
\hline 20 & 0,41 & 0,37 & 0,31 & 0,39 & 0,43 & 0,45 & 0,39 & & 0,47 & & 0,4 & 0,38 & 0,2 & 0,24 & 0,22 \\
\hline 21 & 0,72 & 0,69 & 0,67 & & 0,68 & 0,54 & 0,63 & & 0,6 & & 0,69 & 0,64 & 0,75 & 0,55 & 0,72 \\
\hline 22 & 0,63 & 0,57 & 0,66 & & & 0,51 & 0,67 & & 0,56 & & 0,68 & 0,68 & 0,76 & 0,42 & 0,77 \\
\hline 23 & 0,39 & 0,44 & 0,38 & & & 0,43 & 0,36 & & 0,38 & & 0,4 & 0,42 & 0,39 & 0,38 & 0,38 \\
\hline 24 & 0,62 & 0,6 & 0,62 & & & 0,64 & 0,64 & & 0,58 & & 0,74 & 0,69 & 0,7 & 0,59 & 0,66 \\
\hline 25 & 0,53 & 0,47 & 0,68 & 0,51 & & 0,61 & 0,34 & & 0,62 & 0,6 & 0,67 & 0,66 & 0,58 & & 0,59 \\
\hline 26 & 0,55 & 0,5 & 0,64 & 0,63 & & & & & & & & & & & \\
\hline 27 & 0,72 & & 0,31 & 0,77 & & & & & & 0,66 & 0,57 & 0,63 & & & 0,49 \\
\hline 28 & 0,55 & 0,55 & & 0,69 & 0,46 & 0,49 & & & & 0,64 & 0,7 & 0,64 & 0,66 & 0,62 & 0,61 \\
\hline 29 & 0,22 & & & 0,29 & 0,4 & 0,31 & & & & 0,42 & 0,37 & 0,3 & & & \\
\hline 30 & & 0,46 & 0,43 & 0,51 & 0,49 & & & & & 0,54 & 0,69 & 0,61 & 0,45 & 0,4 & 0,45 \\
\hline 31 & & & 0,57 & 0,73 & & & 0,61 & & & 0,67 & 0,68 & 0,59 & 0,47 & & 0,46 \\
\hline
\end{tabular}

Fuente: elaboración propia

\section{Resultados}

De acuerdo con los resultados de ICA obtenidos, se realizó una discriminación por municipios y se dividió en dos periodos el análisis: del 2008 al 2011, y del 2012 al 2015. A continuación, se presenta el comportamiento de la calidad del agua en cada uno de los municipios de la cuenca del río Bogotá. 


\subsection{Comportamiento ICA municipio de Villapinzón}

La CAR dispuso cuatro puntos de monitoreo en la cuenca alta del río Bogotá que pertenecen al municipio de Villapinzón. La figura 2 evidencia que las categorías "Aceptable" y "Regular" son las de mayor presencia en el periodo 2008-1 a 20112, y ningún periodo alcanza "Buena calidad". El semestre 2008-2 es el semestre con mejor comportamiento con respecto a los otros años de estudio. La estación Puente Villapinzón es el punto con mejor comportamiento en el tiempo estudiado.

La figura 3 muestra el comportamiento entre los periodos 2012-1 a 2015-1. La calidad del agua mejora sustancialmente, en comparación con los semestres que la anteceden. El punto Puente Villapinzón es el punto de monitoreo que registra

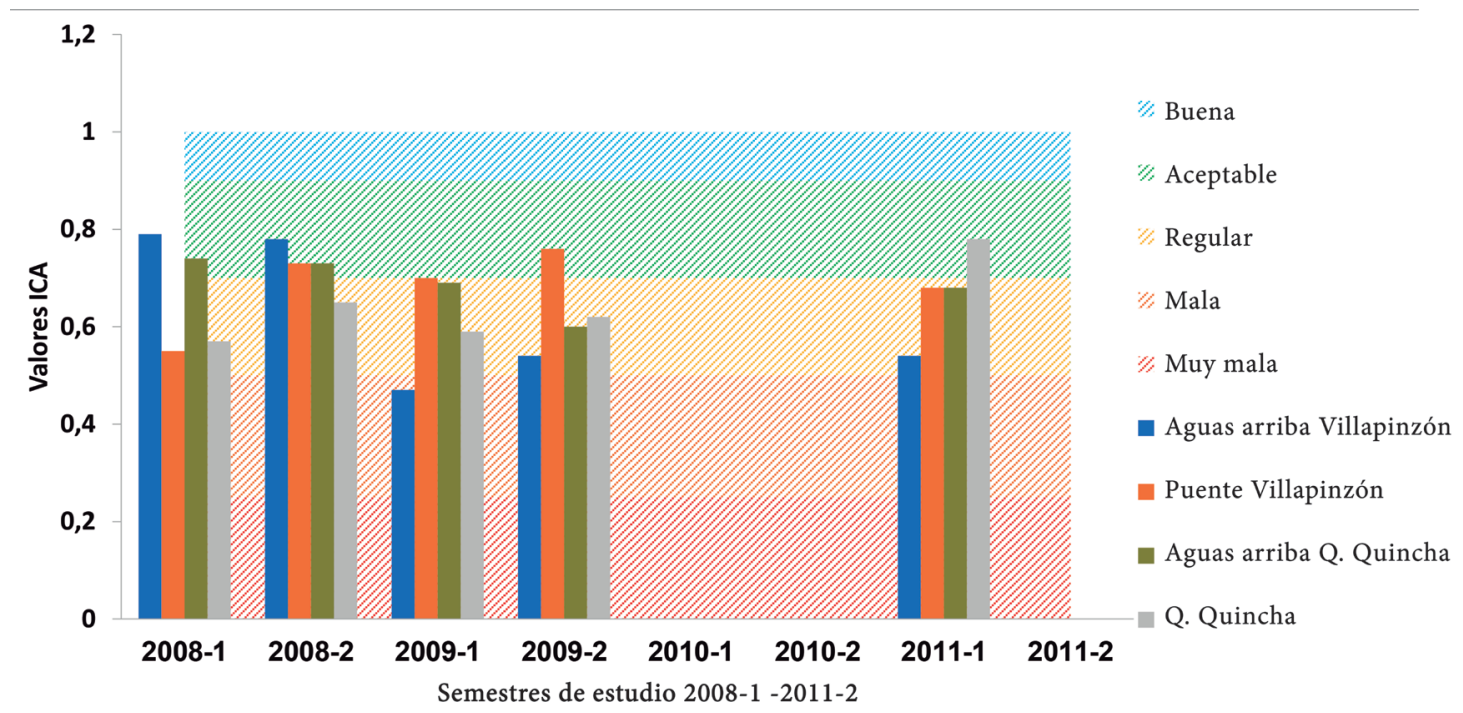

Figura 2. ICA estaciones municipio de Villapinzón 2008-1 a 2011-2

Fuente: elaboración propia

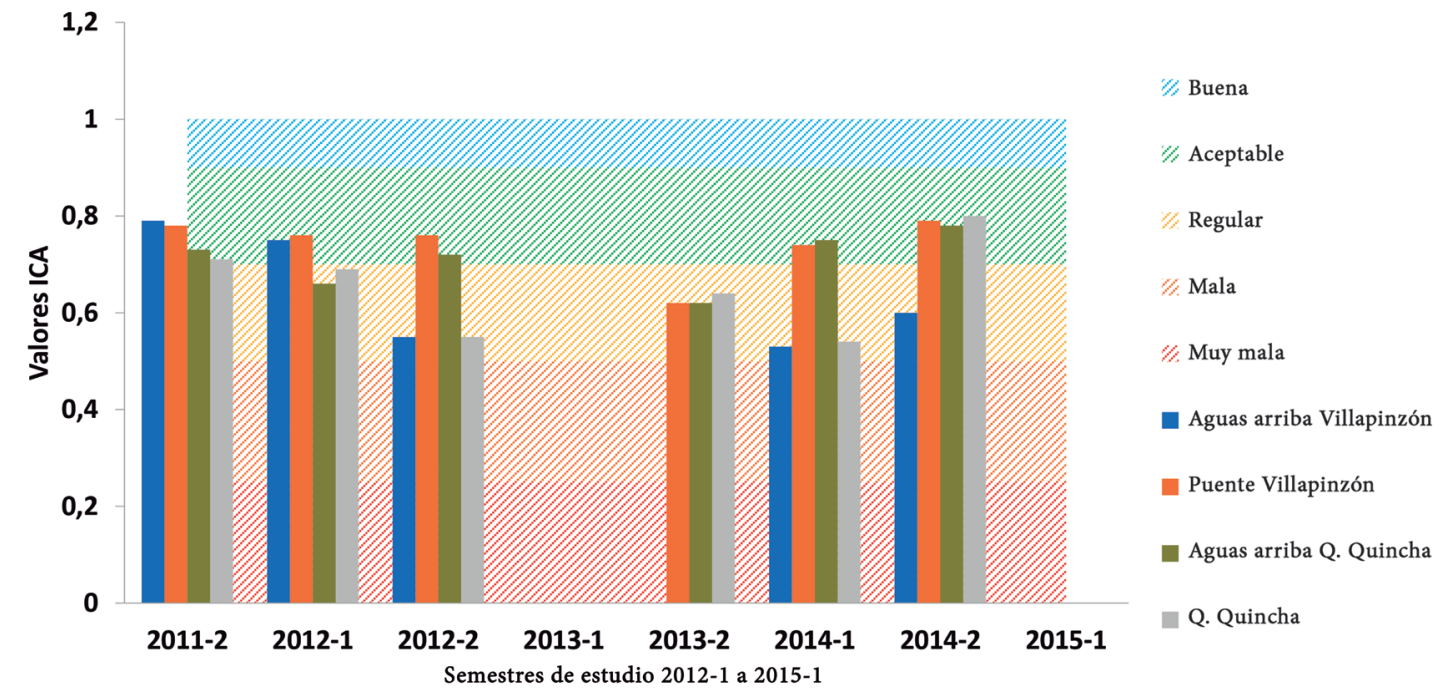

Figura 3. ICA estaciones municipio de Villapinzón 2012-1 a 2015-1

Fuente: elaboración propia 
valor ICA más alto en relación con los demás puntos de monitoreo. No se evidencia categoría "Muy mala" para ninguno de los semestres estudiados, y prevalece "Aceptable" como umbral alcanzado durante la mayor parte del tiempo.

En el municipio de Villapinzón, la categoría "Aceptable" se presenta en tres años: 2008, 2012 y 2015, el mismo tiempo en el que no se presenta información (2010, 2011 y 2013). La clasificación "Regular" se hace presente para los años 2009 y 2014. No se evidencia en ninguno de los años clasificación "Buena" o "Muy mala", como lo muestra la tabla 4.

Tabla 4. Calificación general del ICA anual, municipio de Villapinzón

\begin{tabular}{|c|c|}
\hline Año & Calidad \\
2008 & Aceptable \\
2009 & Regular \\
\hline 2010 & No registra \\
2011 & No registra \\
\hline 2012 & Aceptable \\
\hline 2013 & No registra \\
\hline 2014 & Regular \\
\hline 2015 & Aceptable \\
\hline
\end{tabular}

Fuente: elaboración propia
En los ocho años de análisis, el municipio de Villapinzón presenta una calidad aceptable al inicio, en la mitad y al final del periodo en cuestión. Es importante tener en cuenta que es allí donde nace la cuenca del río Bogotá, y donde se espera una calidad del agua buena. Sin embargo, como indica [7], Villapinzón, Chocontá y San Benito son la cuna industrial del tratamiento de cuero, actividad que hace uso de sustancias químicas tales como mercurio, sulfuros, cloruros y sulfatos, que perjudican la fuente hídrica luego de ser vertidos sin un adecuado tratamiento, pues la actividad se realiza de manera artesanal y no cuenta con mecanismos de tratamiento de agua, como lo resalta [8].

Otro aspecto de igual importancia es la vocación del suelo del municipio. De acuerdo con el Dane [9], Villapinzón registra una alta actividad agropecuaria, principalmente cultivo de papa. Esto ha causado que sus suelos presenten procesos de degradación debido al uso de plaguicidas, fertilizantes y agroquímicos que, mediante efectos de escorrentía, descienden hasta las aguas del río Bogotá.

\subsection{Comportamiento ICA municipio de Chocontá}

La CAR dispuso ocho puntos de monitoreo de la cuenca alta que pertenecen al municipio de Chocontá. La figura 4 muestra que la categoría ICA más representativa durante el periodo 2008-1 a 2011-2 es "Mala"

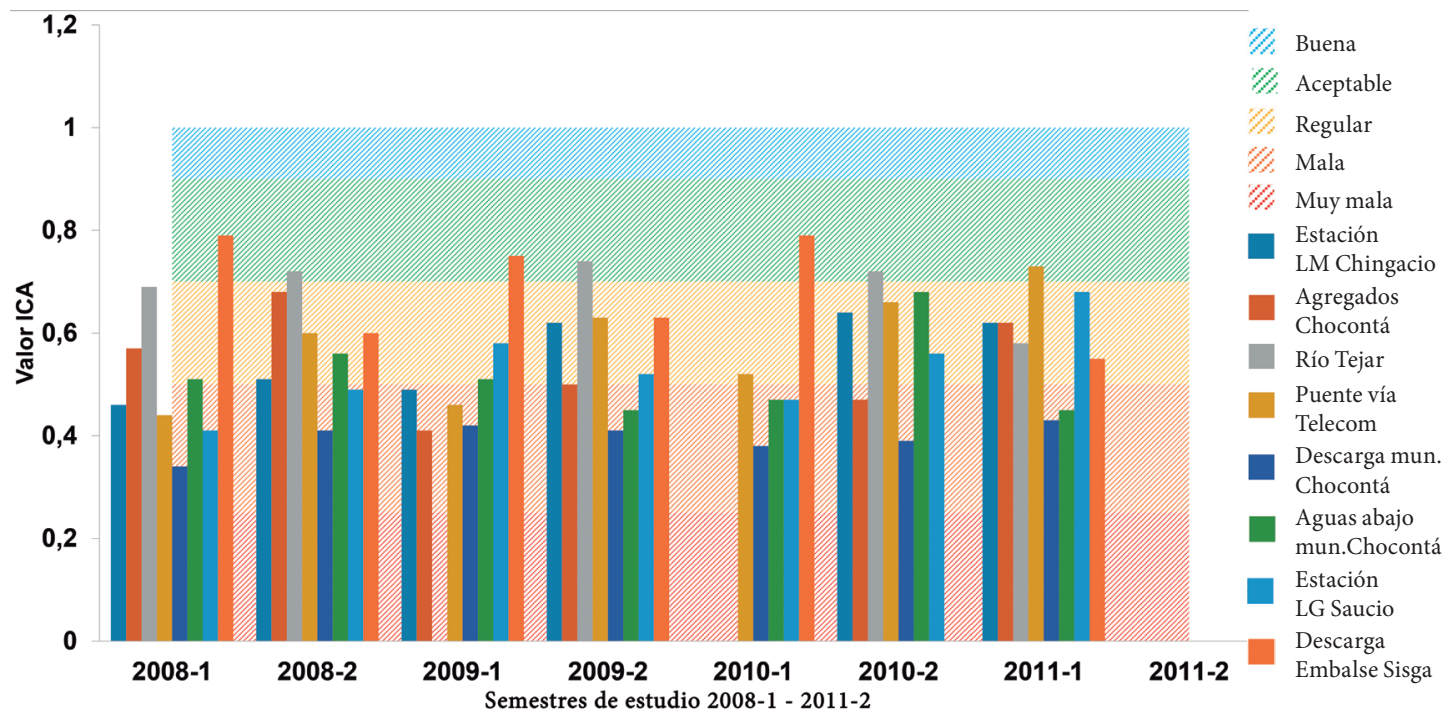

Figura 4. ICA estaciones municipio de Chocontá 2008-1 a 2011-2

Fuente: elaboración propia 


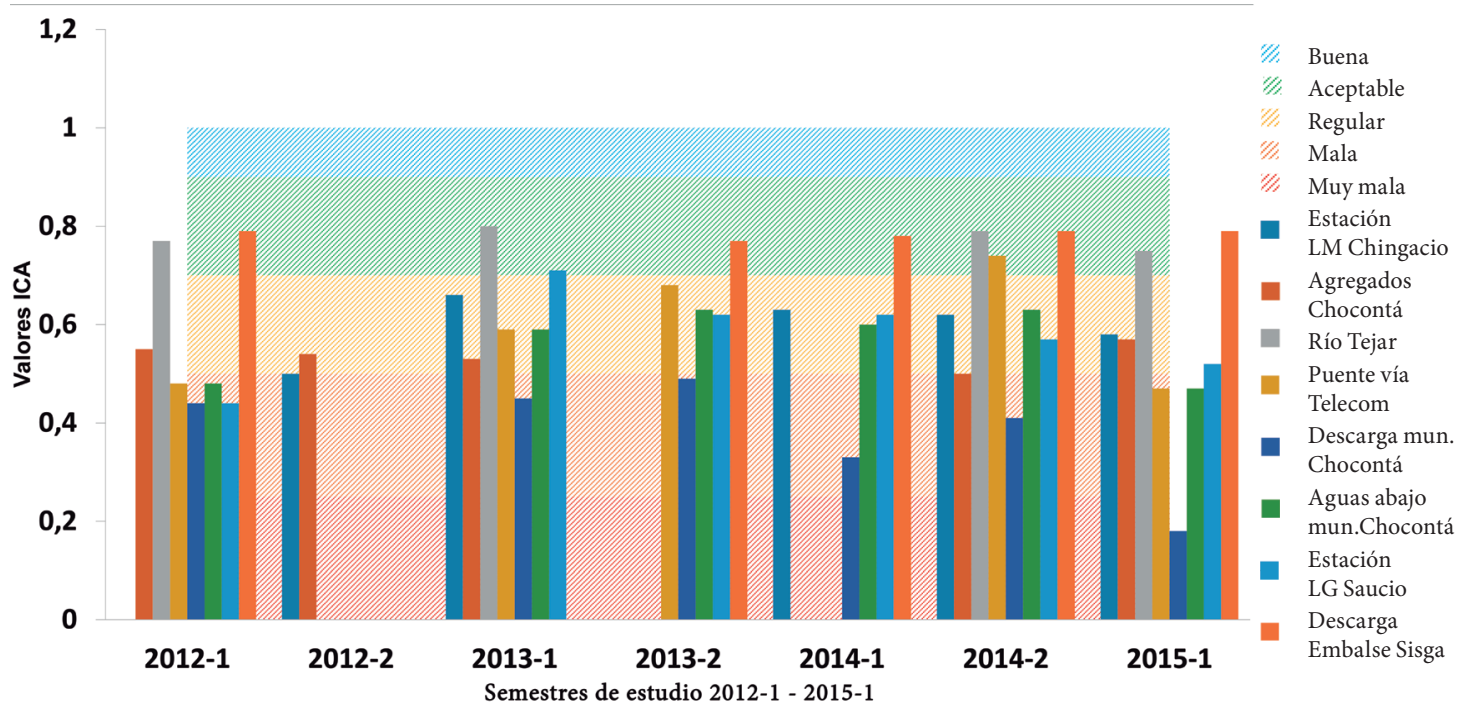

Figura 5. ICA municipio de Chocontá 2012-1 a 2015-2

Fuente: elaboración propia

y “Regular”. La estación de monitoreo del embalse del Sisga es el punto que mejor comportamiento tiene, al presentar una calidad "Aceptable" durante el primer semestre de los años 2008, 2009 y 2010. En ninguno de los semestres estudiados se evidencia que algún punto de monitoreo alcanzó una calidad "Buena".

Duranteel periodo2012-1 a 2015-1, seobserva que en ningún momento los puntos de monitoreo alcanzan una clasificación "Buena". Las estaciones de monitoreo con mejor comportamiento son los correspondientes al Río Tejar y descarga embalse del Sisga, con una calidad de agua "Aceptable" en el periodo analizado.

En el municipio de Chocontá, la categoría ICA más representativa es la "Regular" en los años 2008, 2010, 2013, 2014 y 2015; entre tanto, para el 2009 la calidad es "Mala", como se puede observar en la tabla 5.

Tabla 5. Calificación general del ICA representado en años, municipio de Chocontá

\begin{tabular}{|c|c|}
\hline Año & Calidad \\
\hline 2008 & Regular \\
\hline 2009 & Mala \\
\hline 2010 & Regular \\
\hline 2011 & No registra \\
\hline 2012 & No registra \\
\hline 2013 & Regular \\
\hline 2014 & Regular \\
\hline 2015 & Regular \\
\hline
\end{tabular}

Fuente: elaboración propia
De acuerdo con los datos descritos anteriormente, se evidencia un marcado deterioro de la calidad del agua, debido a la carga contaminante con la que viene desde el municipio de Villapinzón aguas arriba. Esto, sumado a la actividad industrial y agrícola que de allí se deriva, se convierte en un gran problema de contaminación ambiental, en especial por efectos de los vertimientos industriales y agropecuarios al río.

\subsection{Comportamiento ICA municipio de Suesca}

La CAR dispuso seis puntos de monitoreo de la cuenca alta que pertenecen al municipio de Suesca: Estación Lm Santa Rosita, Puente Santander, Descarga municipio de Suesca, Descarga embalse del Tominé, Aguas arriba Descarga Papeles y Molinos. La figura 6 muestra que la categoría "Regular" es la de mayor presencia durante los semestres 2008-1 a 2011-2. La estación de monitoreo Descarga embalse del Tominé es la que mejor calidad presenta durante los periodos de tiempo estudiados.

En el embalse del Tominé, exactamente en su afluente principal, el río Tominé, se encuentran una gran cantidad de macrófitas confinadas, cuyo servicio ambiental es filtrar naturalmente el agua con el fin de depurar impurezas. De esta manera, se logra el objetivo de mejorar la calidad hídrica [10] que en algún momento se pudo ver afectada por el 


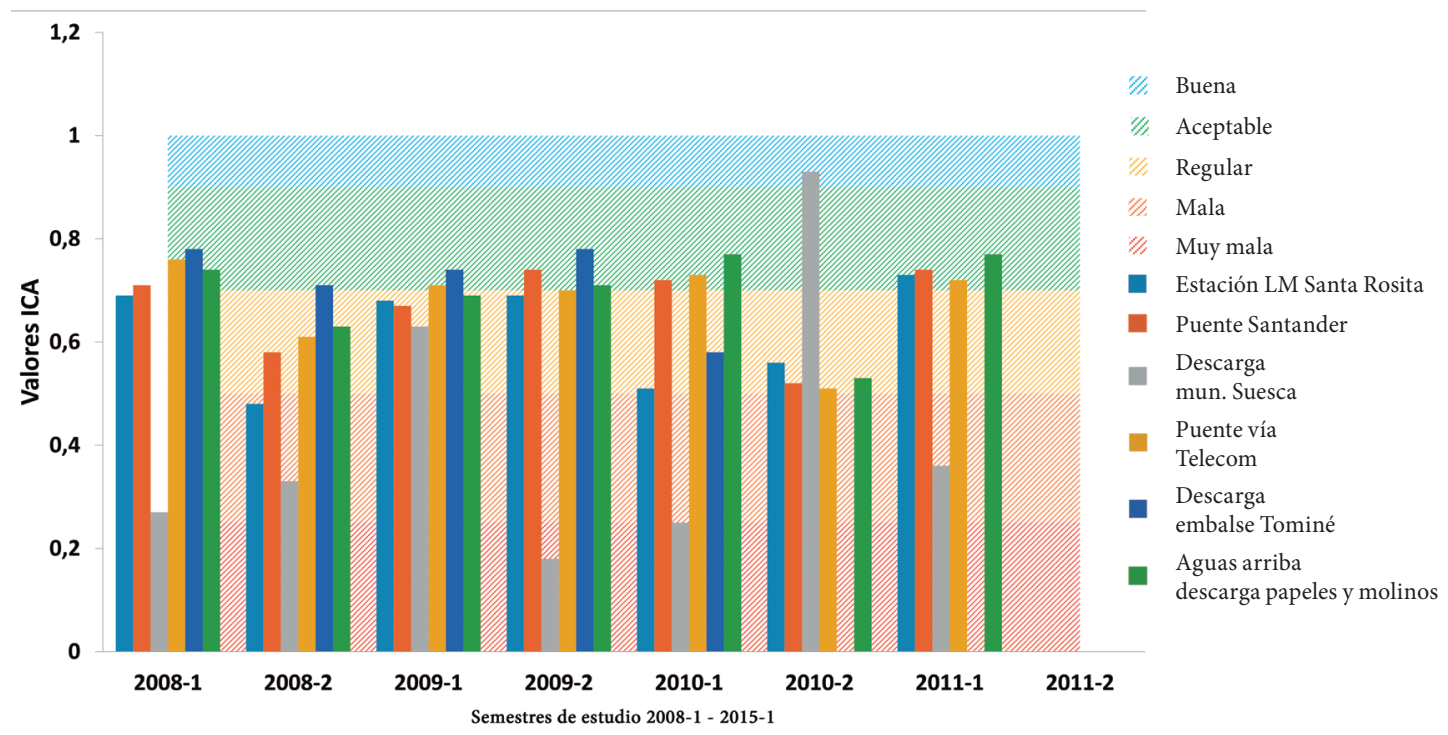

Figura 6. ICA municipio de Suesca 2008-1 a 2011-2

Fuente: elaboración propia

excesivo uso de agroquímicos en otros puntos de la cuenca alta, en razón a las actividades agropecuarias que allí se desarrollan.

En general, entre los semestres 2008-1 a 2011-2, el de mejor comportamiento es el 2008-1, cuyos valores son más altos con respecto a los demás. El mejor resultado obtenido en todos los municipios y estaciones de la cuenca alta del río Bogotá se encuentra en el semestre 2010-2 para la estación Descarga municipio de Suesca, presentándose calidad "Buena".
La figura 7 presenta el comportamiento de los ICA para los semestres de estudio 2012-1 a 2015-1. Los puntos de monitoreo Estación Lm Santa Rosita y Puente Santander, son los puntos de monitoreo con mejor comportamiento con el paso del tiempo, especialmente en los semestres 2013-1, 2014-1 y 2014-2.

La categoría ICA que más se presenta en el municipio de Suesca es "Regular" para cuatro años: 2009, 2010, 2014 y 2015; seguida de calidad "Aceptable" para los años 2008 y 2013. Sin embargo,

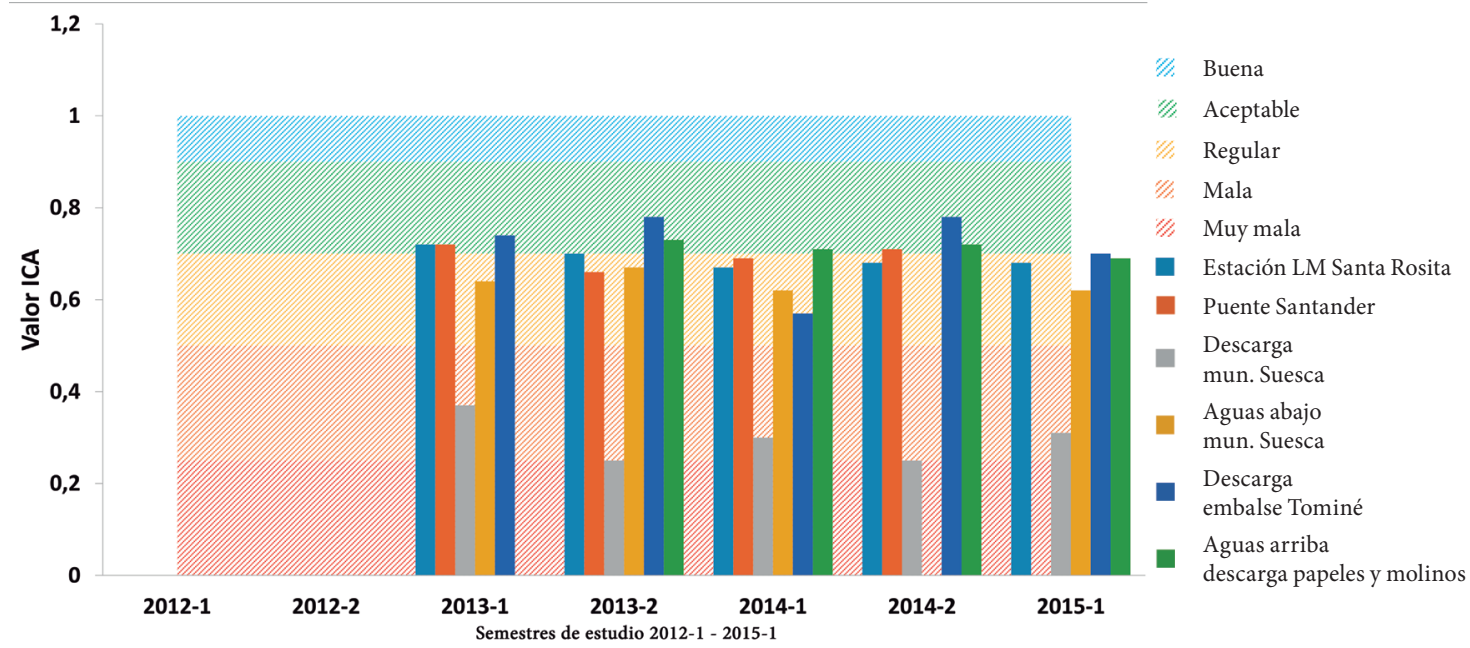

Figura 7. ICA municipio de Suesca 2012-1 a 2015-1

Fuente: elaboración propia 
existen dos años cuyo valor "No registra": 2011 y 2012. A continuación, la tabla 6 muestra el comportamiento anual para el municipio.

Tabla 6. Calificación general del ICA anual, municipio de Suesca

\begin{tabular}{|c|c|}
\hline Año & Calidad \\
\hline 2008 & Aceptable \\
2009 & Regular \\
\hline 2010 & Regular \\
\cline { 2 - 2 } 2011 & No registra \\
\hline 2012 & No registra \\
\hline 2013 & Aceptable \\
\hline 2014 & Regular \\
\cline { 2 - 2 } 2015 & Regular \\
\hline
\end{tabular}

Fuente: elaboración propia

El esquema de ordenamiento territorial (ЕОт) de Suesca [11] menciona dos actividades económicas fundamentales: el desarrollo turístico y la actividad agropecuaria. Asimismo, establece una política ambiental que pacta la protección a las fuentes hídricas y de los ecosistemas páramos como prioridad, debido a la escases del recurso hídrico en la región. Sin embargo, en el Plan de Desarrollo Municipal 20122016 [12], que consta de cuatro ejes programáticos, se destaca el eje 3: "Juntos por Suesca, por la Seguridad, la convivencia y el medio ambiente". Este eje evidencia la importancia de atender las necesidades ambientales en el municipio, pues se ponen de manifiesto conflictos por el uso del agua derivados de las actividades económicas que se desarrollan, tales como minería, agricultura intensiva y ganadería extensiva.
Estas dinámicas industriales repercuten en el cuerpo de agua de manera negativa, lo cual se evidencia en el análisis realizado, pues las aguas correspondientes al municipio de Suesca representan una categoría ICA "Regular" para la mayor parte del periodo de estudio.

\subsection{Comportamiento ICA municipio de Gachancipá}

Los puntos de monitoreo establecidos por la CAR para el municipio de Gachancipá son específicamente tres: Estación lg Pte. Florencia, Descarga municipio Gachancipá, y Aguas abajo municipio Gachancipá. La figura 8 muestra las variaciones de calidad para los periodos 2008-1 a 2011-2; la categoría más representativa durante este periodo de tiempo es "Regular". El punto de monitoreo con mejor comportamiento es el punto Estación lg Pte. Florencia, cuyo valor ICA es más alto con respecto a los otros puntos de monitoreo establecidos para la zona. En ninguno de los periodos de estudio la categoría ICA supera la categoría "Buena".

En la figura 9 podemos evidenciar el comportamiento para los semestres de estudio 2012-1 a 2015-1. La categoría "Regular" es la más común para los semestres estudiados. El punto de monitoreo de mejor comportamiento es Aguas abajo municipio Gachancipá, ya que mantuvo valores en "Regular" y "Aceptable"; entre tanto, el de comportamiento menos favorable es Descarga municipio de Gachancipá, que en los últimos tres semestres de análisis desmejoró su calidad llegando a "Muy mala".

En el municipio de Gachancipá, la clasificación ICA que más se presenta es la "Regular" para los años 2009, 2010, 2011, 2013 y 2014. Para los años 2008 y 2015 predomina la calidad "Aceptable",

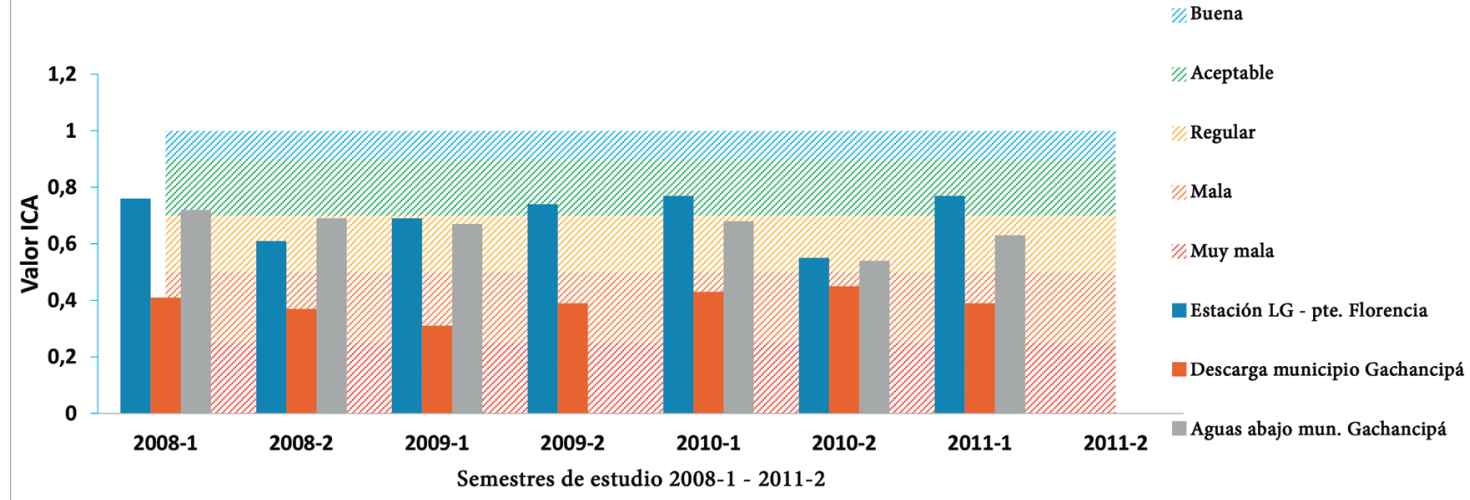

Figura 8. ICA municipio de Gachancipá 2008-1 a 2011-2

Fuente: elaboración propia 


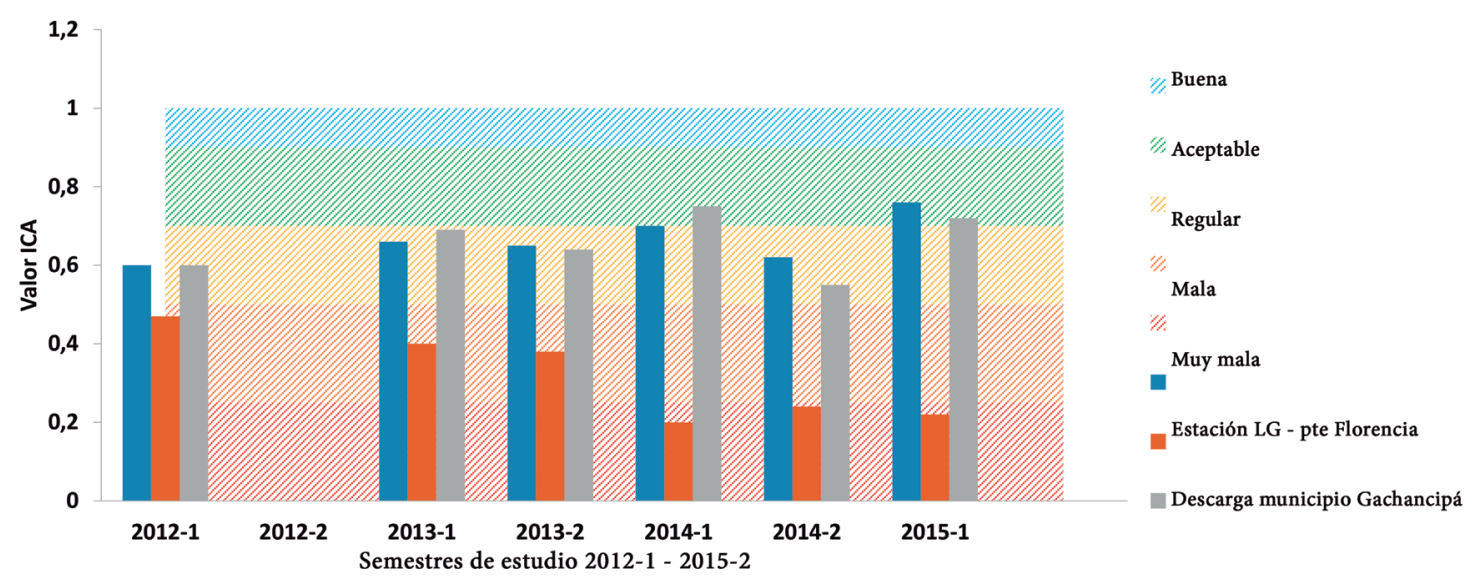

Figura 9. ICA municipio de Gachancipá 2012-1 a 2015-1

Fuente: elaboración propia

mientras que para el 2012 no se registran datos; la tabla 7 muestra un resumen de lo descrito.

Tabla 7. Calificación general del ICA anual, municipio de Gachancipá

\begin{tabular}{|c|c|}
\hline Año & Calidad \\
\hline 2008 & Aceptable \\
\hline 2009 & Regular \\
\hline 2010 & Regular \\
\cline { 2 - 2 } 2011 & Regular \\
\hline 2012 & No registra \\
\hline 2013 & Regular \\
\hline 2014 & Regular \\
\cline { 2 - 2 } 2015 & Aceptable \\
\cline { 2 - 2 }
\end{tabular}

Fuente: elaboración propia

El Plan de Desarrollo Municipal del municipio de Gachancipa [13], definido para el periodo 2012-2015, establece que el principal eje hídrico que cruza el municipio de Gachancipá de norte a sur es el río Bogotá, e indica que estas aguas abastecen gran cantidad de cultivos de flores que se encuentran en la región.

La auditoría especial a la gestión sobre el río Bogotá [14], realizada por la Contraloría General de la Nación en el 2013, afirma que existen serias problemáticas ambientales en torno al agua del río Bogotá en este tramo, e incluye: inadecuados vertimientos de aguas servidas, tanto agrícolas, como domésticas y comerciales; inapropiada disposición de residuos sólidos; deforestación de zonas protegidas y zonas de ronda de las áreas hídricas del río por habitantes; algunos cultivos ilegales; e ineficiente gestión de contaminantes. Estos aspectos conllevan a una serie de impactos negativos en torno al agua que, sin duda, perjudican la calidad del río Bogotá, cuestión evidente en los resultados arrojados por el estudio.

\subsection{Comportamiento ICA municipio de Tocancipá}

Cuatro puntos de monitoreo ubicados por la CAR para la cuenca alta del río, hacen parte del municipio de Tocancipá. La figura 10 muestra las variaciones en calidad que ha tenido el municipio entre los periodos 2008-1 a 2011-2, cuyos valores ICA muestran que "Regular" es la categoría más representativa. No se cuenta con información registrada para los semestres 2010-1 y 2011-2. El municipio no cuenta con categoría "Aceptable" o "Buena" en ningún periodo histórico. Asimismo, ningún punto de monitoreo alcanzó la línea que indica calidad "Aceptable", como tampoco algún punto indica tener "Muy mala calidad".

La figura 11 muestra el comportamiento de calidad en los semestres 2012-1 a 2015-1 para el municipio de Tocancipá. La estación de monitoreo más estable es Descarga municipio Tocancipá, la cual no mostró variaciones significativas con el paso del tiempo, manteniéndose en una calidad "Mala". En los periodos 2014-1 y 2015-1 se mantienen los valores para cada una de las estaciones del municipio, sin embargo, al compararlo con el mismo periodo en años anteriores, se observa una mejoría en la calidad del agua.

En esta zona de estudio la calidad del agua que más predomina es "Regular", con una incidencia de cinco años: 2008, 2009, 2010, 2012 y 2015. Entre 


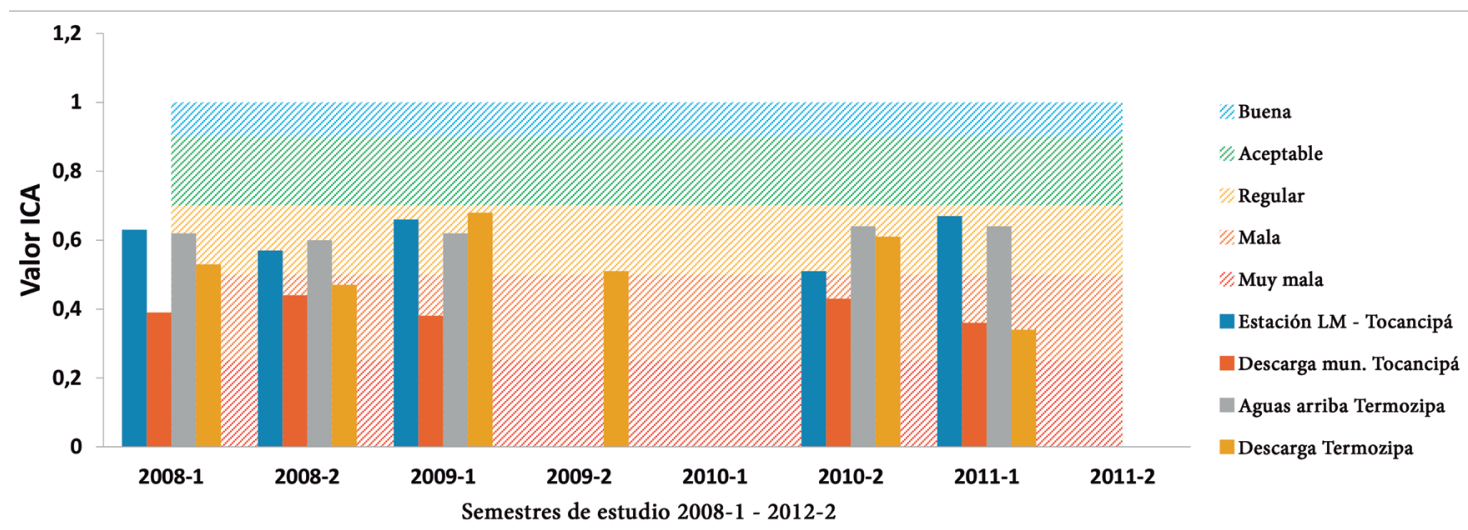

Figura 10. ICA municipio de Tocancipá 2008-1 a 2011-2

Fuente: elaboración propia

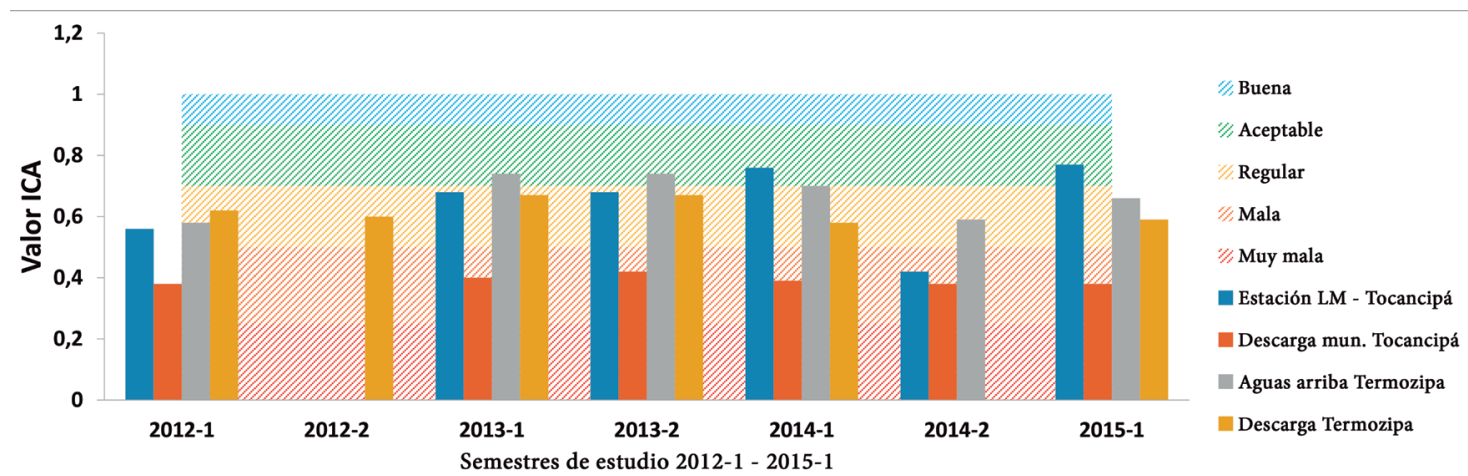

Figura 11. ICA municipio de Tocancipá 2012-1 a 2015-1

Fuente: elaboración propia

tanto, solo uno de los ocho años se enmarca en la categoría "Aceptable". El 2011 no cuenta con información registrada para el segundo.

Tabla 8. Calificación general del ICA anual, municipio de Tocancipá

\begin{tabular}{|c|c|}
\hline Año & Calidad \\
\hline 2008 & Regular \\
\hline 2009 & Regular \\
\hline 2010 & Regular \\
\hline 2011 & No registra \\
\hline 2012 & Regular \\
\hline 2013 & Aceptable \\
\hline 2014 & Mala \\
\hline 2015 & Regular \\
\hline
\end{tabular}

Fuente: elaboración propia

\subsection{Comportamiento ICA municipio de Zipaquirá}

La figura 12 muestra el comportamiento que ha tenido el municipio de Zipaquirá durante los semestres 2008-1 a 2011-2. Es evidente que ningún punto de monitoreo alcanza la categoría "Buena"; el índice se mantiene entre "Mala" y "Regular". En el punto conocido como Río Negro, en el semestre 2008-1, disminuye su calidad al ubicarse en la franja roja que indica calidad "Muy mala". La estación río Neusa presenta calidad "Aceptable" en los semestres 2008-1 y 2009-2. El semestre que refleja mejor calidad en las aguas es 2009-2, en todos los puntos de estudio.

Durante el periodo 2012 a 2015, se evidencia que en ningún momento el ICA alcanza una calidad "Aceptable". El indicador muestra que la calidad del agua permanece entre "Regular" y "Mala", dependiendo de su ubicación y el momento del tiempo. 


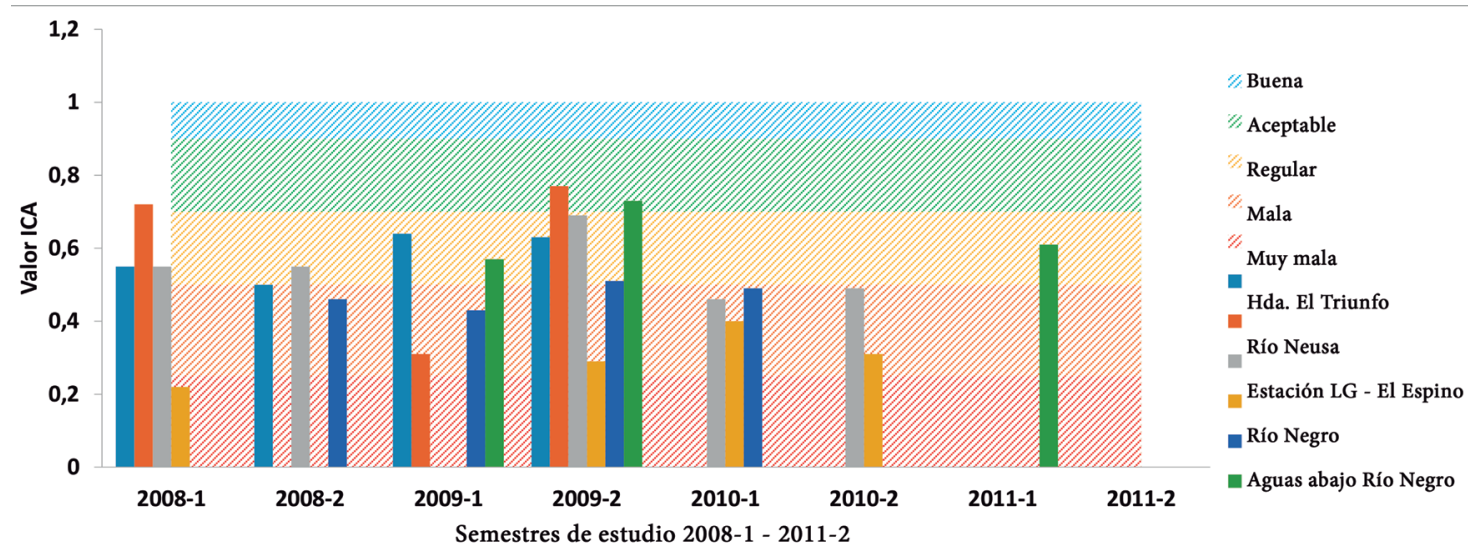

Figura 12. ICA municipio de Zipaquirá 2008-1 a 2011-2

Fuente: elaboración propia

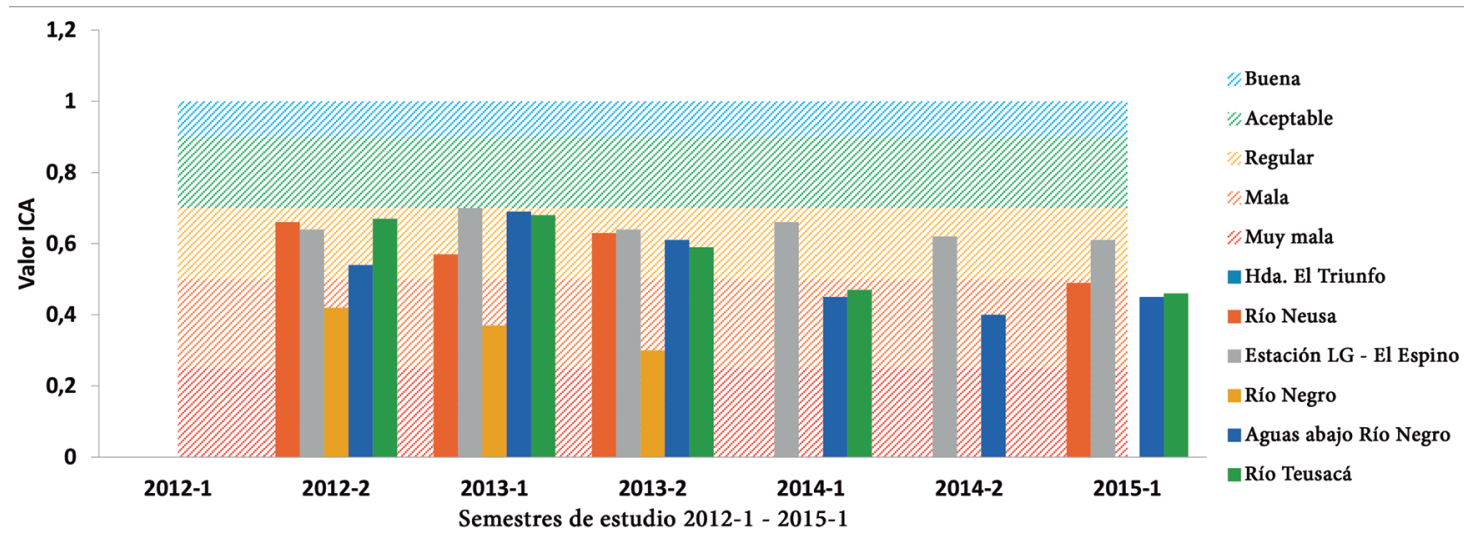

Figura 13. ICA municipio de Zipaquirá 2012-1 a 2015-1

Fuente: elaboración propia

En la zona de estudio, la categoría ICA más frecuente es "Regular", presente en los años 2009, 2013 y 2014; existen tres años sin información: 2008, 2011 y 2012, y para efectos del 2010 y el 2015, la categoría es "Mala" (ver tabla 9).

Tabla 9. Calificación general del ICA anual, municipio de Zipaquirá

\begin{tabular}{|c|c|}
\hline Año & Calidad \\
\hline 2008 & No registra \\
\hline 2009 & Regular \\
\hline 2010 & Mala \\
\hline 2011 & No registra \\
\hline 2012 & No registra \\
\hline 2013 & Regular \\
\hline 2014 & Regular \\
\hline 2015 & Mala \\
\hline
\end{tabular}

Fuente: elaboración propia
El semestre 2011-2 y gran parte del semestre 2012-2 no tienen información registrada para realizar procesamiento de datos y obtener los ICA, aspecto que repercutió en toda la cuenca, lo cual impidió realizar un seguimiento detallado del comportamiento de calidad para cada municipio que pertenece a la cuenca alta.

\section{Discusión y conclusiones}

Se espera que el nacimiento de un río presente, en sus primeros kilómetros de recorrido, una calidad de agua buena. Sin embargo, el río Bogotá en su cuenca alta presenta una calidad aceptable en los puntos ubicados en Villapinzón y Chocontá, decayendo a medida que avanza: en las estaciones correspondientes a los municipios de Suesca y Gachancipá presenta una calidad regular, luego una calidad mala en el municipio de Tocancipá. Los factores 
antrópicos son la principal causa de contaminación y deterioro ambiental, en razón a las actividades que se desarrollan en torno a sus aguas.

Como se indica en los resultados, la principal actividad económica de los municipios de Villapinzón (nacimiento del río Bogotá) y Chocontá son las curtiembres, proceso que genera una cantidad considerable de vertimientos con una alta carga orgánica y de cromo, principalmente, impidiendo que los rasgos de calidad del agua se mantengan en el margen de aceptabilidad.

Otra actividad a tener en cuenta en el área es la agricultura, común denominador para los municipios ubicados en la sabana cundiboyacense (área de estudio), pues como consecuencia del uso de agroquímicos, sumado a los procesos naturales de escorrentía e infiltración, es posible que trazas de ellos lleguen a la fuente; evidencia de lo anterior es la calificación ICA "Regular" y "Mala" para la mayor parte de la cuenca alta.

Un efecto que adicionalmente afecta la calidad del recurso es la rápida industrialización que ha sufrido la región. Ejemplo de esto es el municipio de Tocancipá, el cual ha concentrado en su territorio industrias de diferentes sectores económicos (automotriz, textiles, manufactura, agroindustria y servicios, entre otros), bajo la figura de zonas francas y parques industriales. Esto significa que movimientos poblacionales se ubiquen o no definitivamente en el municipio, además de un evidente aumento en la demanda del recurso hídrico.

Se analizan los planes de ordenamiento territorial y los planes de desarrollo de los municipios, y si bien se menciona la importancia del río Bogotá en su área, es de vital importancia que a la hora de abordar la formulación de estos instrumentos de gestión, se dé un mayor protagonismo al tema ambiental en aras de atender los problemas de uso y de contaminación que presenta el río Bogotá en esta parte de la cuenca.

Los resultados obtenidos evidencian la necesidad de formular nuevas estrategias ambientales, o reformular las existentes, en el propósito de cuidar y preservar la fuente hídrica desde su nacimiento, pues a medida de que el tramo avanza, la calidad del agua disminuye al punto de restringir su uso a demandas industriales.

El acceso restringido a cierto tipo de información de carácter técnico por parte de algunas entidades ambientales que manejan la información relacionada con la calidad de agua del río Bogotá, se convirtió en una limitante, como también la omisión de algunos parámetros específicos para algunos indicadores relevantes de los datos que se encuentran disponibles.

\section{Agradecimientos}

A la Universidad Cooperativa de Colombia y a la Universidad Distrital Francisco José de Caldas, por apoyar el desarrollo académico y respaldar el proceso de investigación.

\section{Referencias}

[1] WWAP, Informe de las Naciones Unidas sobre el Desarrollo de los Recursos Hídricos en el Mundo 2016: Agua y Empleo, París, unEsco, 2016. Disponible en: http:// unesdoc.unesco.org/images/0024/002441/244103s.pdf.

[2] M. F. Castro-Fernández, J. Almeida, J. Ferrer y D. M. Díaz-Casallas, "Indicadores de la calidad del agua : evolución y tendencias a nivel global," Ingeniería Solidaria, vol. 10, n. ${ }^{\circ} 17$, pp. 111-124, en.-dic. 2014. doi: http://dx.doi.org/10.16925/in.v9i17.811.

[3] Ideam, El Medio Ambiente en Colombia, 2a ed., Bogotá, 2001. Disponible en: http://documentacion.ideam. gov.co/openbiblio/bvirtual/000001/000001.htm.

[4] C. P. Guzmán, "Procesos de monitoreo a la calidad hídrica del río Bogotá realizados por la Corporación Autónoma Regional de Cundinamarca-CAR," Pontificia Universidad Javeriana, 2010. Disponible en: https://repository.javeriana.edu.co/bitstream/handle/10554/732/eam51.pdf? sequence $=1$.

[5] CAR, "Boletín de Calidad de las Cuencas de la Jurisdicción CAR-2011," Bogotá, 2011. Disponible en: https://www.car.gov.co/index.php?idcategoria=32981.

[6] Ideam, "Hoja metodológica del indicador Índice de calidad del agua," Sistema de Indicadores Ambientales de Colombia-Indicadores de Calidad del agua superficial, p. 10, 2011.

[7] L. A. Artuz, M. S. Martínez y C. J. Morales, "Las industrias curtiembres y su incidencia en la contaminación del río Bogotá", ISOCUANTA, vol. 1, no. 1, 2011. Disponible en: http://revistas.usta.edu.co/index.php/isocuanta/article/view/1387

[8] L. Ardila \& K. Cortés, "El Ecoparque Industrial como parte de la solución para la recuperación del río Bogotá", Plaza Capital-Universidad del Rosario, Bogotá, Oct-2014. Disponible en: http://www.urosario.edu.co/Plaza-Capital/Nacion/El-Ecoparque-Industrial-como-parte-de-la-solucion/. 
[9] Departamento Administrativo Nacional de Estadística DANE, "Censo Experimental Municipio de Villapinzón,” 2001. Disponible en: http://www.dane.gov. co/files/investigaciones/agropecuario/enda/ena/ censo_papa_villapinzon.pdf.

[10] Empresa de Energía de Bogotá-Eeb, "Plan de Manejo Ambiental (PMA) del Embalse de Tominé".

[11] Alcaldía Municipal de Suesca, "Esquema de Ordenamiento Territorial Municipio Suesca, Documento Soporte Técnico-Formulación”, 2014.

[12] Alcaldía de Suesca, "Plan de Desarrollo Municipal," 2012.
[13] Alcaldía de Gachancipá, Acuerdo N.o 011 de 2012 Adóptese el Plan de Desarrollo Municipal "Progreso para todos” 2012-2015 del municipio de Gachancipá Cundinamarca, 2012.

[14] Contraloría General de la República, “Auditoría especial a la gestión sobre el Río Bogotá-Intersectorial y articulada," 2014. Disponible en: https://www.minsalud.gov.co/sites/rid/Lists/BibliotecaDigital/RIDE/ $\mathrm{DE} / \mathrm{OCI} /$ auditoria-especial-a-la-gestion-rio-bogota.pdf. 